\title{
Internal carotid artery dissection after laparoscopic surgery
}

Author(s): Martín-Mateos E.' , Gómez-Ríos M.Á.' , Casans Francés R. , Freire-Vila E.'

Institute(s): ' Complejo Hospitalario Universitario A Coruña, Dept of Anaesthesiology \& Intensive Care, A Coruña, Spain,

${ }^{2}$ Hospital Clinico Universitario 'Lozano Blesa', Dept of Anaesthesiology \& Intensive Care, Zaragoza, Spain

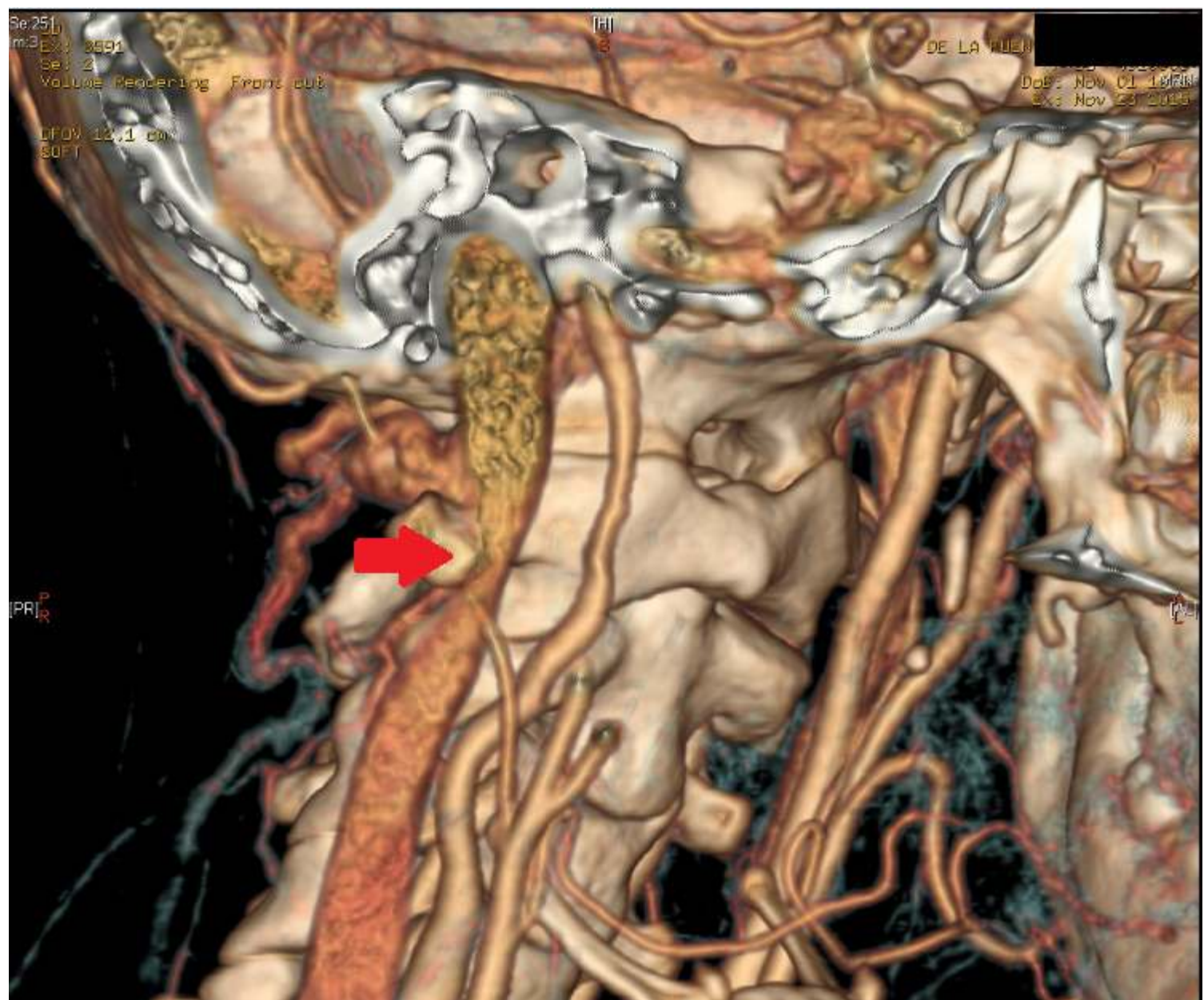

Background: Headache is a common symptom. However, more serious, uncommon and life-threatening conditions as carotid artery dissection (CAD) can be associated with severe neurologic sequelae in otherwise young, healthy patients. Clinicians should be familiar with the presentation and management strategies for this complication.

Case report: The patient was a $39-y e a r-o l d, 58 \mathrm{~kg}$, female, without relevant medical history. She was scheduled for laparoscopic right adnexectomy due to mucinous cystadenoma. Induction and maintenance of anaesthesia were uneventful. The procedure was carried out without incident, and the patient's trachea was extubated at the end of the operation, with minimal hemodynamic fluctuation. In the postanaesthesia care unit, the patient developed a persistent oppressive rightsided frontotemporal headache and neck pain with swallowing, partial ptosis and reactive miosis without visual loss. Contrast angiography revealed a spontaneous CAD. Anticoagulation and antiplatelet therapy were initiated. Our patient was discharged home without any neurologic sequelae.

Discussion: CAD is a rare condition. Its aetiology is either spontaneous or traumatic, although simple manipulation of the neck can evoke a dissection. Several factors may have contributed. The combination of pneumoperitoneum along with the steep Trendelemburg position affect cerebrovascular and hemodynamic homeostasis increasing the risk of arterial dissections, neck hyperextension during endotracheal intubation could have played a role; however Totaltrack device allowed an ETI with the head in a neutral position.

The classic triad of unilateral pain in the head, face or neck, partial Horner's syndrome and cerebral ischemia is found in fewer than $30 \%$ of cases. Cranial nerve palsies and pulsatile tinnitus have been reported. The gold standard for diagnosis of CAD is contrast angiography. Treatment of CAD consists of avoiding or limiting neurological deficit through the prevention of thrombus formation or associated embolism. Therefore, anticoagulation and antiplatelet therapy are the mainstay of the treatment.

References: Ortiz J, Ruland S. Cervicocerebral artery dissection. Curr Opin Cardiol 2015;30:603- 10.

Possible aetiology of headache:

Dehydration

Sleep deprivation

Intentional or inadver-tent dural puncture during a neuraxial anesthesia technique

Inhaled anesthetic agent

Specific surgical procedures

Others

Risk factors associated with carotid artery dissection:

\section{Genetic factors}

Marfan syndrome

Ehlers-Danlos syndrome-type IV

Osteogenesis imperfecta-type I

Fibromuscular dysplasia

Hyperhomocysteinemia

Hemochromatosis

Alfa I-Antitrypsin deficiency

Turner syndrome

Down síndrome

Williams síndrome

Menkes disease

Moyamoya disease

Polycystic kidney disease

Pseudoxanthoma elasticum

\section{Acquired and anatomic factors}

Cervical trauma

latrogenic after surgical interventions

Medical procedures

Oropharyngeal infections

Arterial hypertension

Vascular tortuosity

Aorta coarctation

Illicit drug abuse

Chiropractic cervical manipulation

Severe exercise

Vomiting

Violent coughing or sneezing

Migraine 